\title{
Micropropagation of Different Citrus Rootstocks Using WPM Medium Culture
}

\author{
Reisane Teles Santiago ${ }^{1}$, Karen Cristina Fialho dos Santos ${ }^{2}$, Carlos Alberto da Silva Ledo ${ }^{2}$, \\ Abelmon da Silva Gesteira ${ }^{2}$, Walter dos Santos Soares Filho ${ }^{2}$ \& Antônio da Silva Souza ${ }^{2}$ \\ ${ }^{1}$ Center for Agrarian Environmental and Biological Sciences, Federal University of Recôncavo da Bahia, Cruz \\ das Almas, Bahia, Brazil \\ ${ }^{2}$ Embrapa Cassava and Fruits, Cruz das Almas, Bahia, Brazil \\ Correspondence: Antônio da Silva Souza, Embrapa Cassava and Fruits, Cruz das Almas, Bahia, Brazil. Tel: \\ 55-3312-8154. E-mail: antonio.silva-souza@embrapa.br
}

Received: November 30, 2018

Accepted: January 8, 2019

Online Published: March 15, 2019

doi: $10.5539 /$ jas.v11n4p136

URL: https://doi.org/10.5539/jas.v11n4p136

\begin{abstract}
Micropropagation is a method that enables in vitro cloning of cultivated plants, irrespective of their potential to produce seeds and their polyembryony rate, guaranteeing genetic identity and absence of phytosanitary problems of the propagated plants if the multiplication process is conducted suitably. In this respect, citrus rootstocks of agronomic interest were micropropagated in Wood Plant Medium (WPM). The experiment was conducted in the municipality of Cruz das Almas, in the Recôncavo Baiano region of Bahia state, Brazil, with 10 rootstocks: 'Indio', 'Riverside' and 'San Diego' citrandarins, 'Sunki Tropical' mandarin, and the hybrids RL $\times$ TR-001, FRL $\times$ $(\mathrm{RL} \times \mathrm{TR})-005, \mathrm{CSM} \times(\mathrm{RL} \times \mathrm{TR})-059, \mathrm{TRH}-051, \mathrm{TRH}-069$ and CSM $\times$ TRBK-Colombia. Nodal segments of these genotypes, with approximate length of $1 \mathrm{~cm}$, were used. The number of plants formed per explant was evaluated 180 days after inoculation. The experimental design was completely randomized, with ten treatments and 12 repetitions, the data obtained were submitted to analysis of variance (F-test) and the grouped means were analyzed by the Scott-Knott test, in both cases a 5\% probability. In vitro culture of explants of the ten genotypes studied presented positive responses to micropropagation in the WPM medium, once the explants of all genotypes showed direct regeneration and formed plants. The citrandarin 'Indio' he stood out with the highest average of explants by plant (4.20).
\end{abstract}

Keywords: in vitro culture, explants, Poncirus hybrids, wood plant medium

\section{Introduction}

In commercial citrus orchards, rootstocks are generally propagated from seeds. But depending on the seeds' polyembryony rate, this multiplication route can impair the genetic fidelity of the rootstock variety to be used, and consequently the uniformity of the groves, since the frequency of zygotic embryos in seeds with low polyembryony rates is usually high, implying restricted formation of nuclear embryos, the target of multiplication since they have the same genetic constitution as the mother plant (Sharma, Prakash, \& Tele, 2009; Soares Filho, Souza, Ledo, Santana, \& Passos, 2014). Besides this, some rootstock varieties produce fruits with low quantities of seeds, such as the Sunki mandarin [Citrus sunki (Hayata) hort. ex Tanaka], hindering its propagation.

Micropropagation is a method that allows avoiding these problems, and can be employed on a large scale for in vitro cloning of rootstocks, regardless of the potential for producing seeds and their polyembryony rate, guaranteeing genetic identity and absence of phytosanitary problems of the propagated plants when performed appropriately (Engelmann, 2011).

In citrus, as is the case of many other plant species groups, the success of any in vitro regeneration system depends on various factors, such as the incubation conditions, choice of culture medium, application of growth regulators and their concentrations, and type of explant. The genotype also has a strong influence on the tissue culture results (Cervera, A. Navarro, L. Navarro, \& Peña, 2008; Tallón, Porras, \& Pérez, 2013).

The majority of studies of in vitro culturing have focused on the effects of growth regulators on the plants, with the influence of the base culture medium having been less studied. Nevertheless, the chemical composition of the 
culture medium is among the most important factors for the success of tissue culturing (Ruzic, Saric, Cerovic, \& Culafic, 2004).

Generally, the production of citrus rootstocks occurs through sowing in nurseries, and depending on the polyembryony rate of the seeds, this route of propagation can compromise the genetic fidelity and uniformity of the orchards, since the zygotic embryos do not preserve the characteristics of the rootstock of interest (Sharma et al., 2009). In addition, some rootstocks do not produce or exhibit a low amount of seed; others are monoembryonic; or have a low germination rate, thus compromising the production of seedlings of certain genotypes.

Wood Plant Medium (WPM) is a basic cultivation medium that has been used for the in vitro establishment of Citrus Germplasm Active Bank of the Embrapa Cassava and Fruits, as it provides very promising results in the multiplication of several citrus genotypes (Carvalho, Souza, Santos, Soares Filho, Ledo, \& Souza, 2016). The objective of the work was to propagate rootstocks of agronomic interest by means of in vitro cultivation using the WPM culture medium.

\section{Materials and Methods}

The experiment was conducted in the municipality of Cruz das Almas, located in the Recôncavo Baiano, which has geographical coordinates $12^{\circ} 40^{\prime} 19^{\prime \prime}$ south latitude, $39^{\circ} 06^{\prime} 23^{\prime \prime}$ west longitude, with an altitude of $226 \mathrm{~m}$.

Developed with the support of Plant Tissue Culture Laboratory of Embrapa Cassava and Fruits, considering 10 rootstocks: 'Indio', 'Riverside' and 'San Diego' citrandarins [C. sunki $\times$ Poncirus trifoliata (L.) Raf.], 'Sunki Tropical' mandarin, and hybrids RL $\times$ TR-001, FRL $\times(\mathrm{RL} \times \mathrm{TR})-005, \mathrm{CSM} \times(\mathrm{RL} \times \mathrm{TR})-059, \mathrm{TRH}-051$, TRH-069 and CSM $\times$ TRBK Colombia, where $\mathrm{RL}=$ 'Rangpur' lime (C. limonia Osbeck), TR $=$ P. trifoliata, $\mathrm{FRL}$ $=$ 'Florida' rough lemon $(C$. jambhiri Lush. $), \mathrm{CSM}=$ common 'Sunki' mandarin, $\mathrm{TRH}=$ trifoliate hybrid, involving $P$. trifoliata, and TRBK $=P$. trifoliata 'Benecke'.

To obtain the explants, five ripe fruits were collected of each genotype. Were used 12 seeds per genotype. The seeds were removed, washed for removal of the mucilage and kept to dry on paper towel, on the laboratory bench at environment temperature for one day. Then the external tegument (testa) was removed and the seeds were disinfested in a laminar flow chamber through immersion in $70 \%$ ethanol for five minutes followed by immersion in a $0.5 \%$ sodium hypochlorite solution containing two droplets of Tween ${ }^{\circledR}$ for 20 minutes, after which they were washed three times in autoclaved distilled water. The seeds were inoculated in tubes containing approximately $10 \mathrm{~mL}$ of WPM in its basal composition. The tubes were kept in a growth room at a temperature of $27 \pm 1^{\circ} \mathrm{C}$, with photon flux density of $30 \mu \mathrm{mol} \mathrm{m} \mathrm{s}^{-2} \mathrm{~s}^{-1}$ and photoperiod of 16 hours.

After 120 days of cultivation were obtained matrix plants, the number of plants obtained varied according to the genotype. The seedlings, with heights ranging from 10 to $12 \mathrm{~cm}$, were used to obtain the explants and establish the experiment. For that purpose, nodal segments with approximate length of $1 \mathrm{~cm}$ were inoculated in glass flasks $(250 \mathrm{~mL})$ containing $50 \mathrm{~mL}$ of WPM, ranging from one to five explants per plant matrix obtained. Then the flasks were taken to the growth room and kept under the same conditions mentioned previously.

At 150 days after inoculation in the culture medium, the explants were subcultured in new medium. The number of plants formed per explant was then evaluated 180 days after inoculation of the nodal segments.

The experimental design was completely randomized, with ten treatments and 12 repetitions, the data obtained were submitted to analysis of variance (F-test) and the grouped means were submitted to the Scott-Knott test, in both cases at $5 \%$ probability. The statistical tests were performed with the SAS program (Statistical Analysis System, version 7.0).

Since the explants came from individuals obtained from germination of seeds, was determined the polyembryony rates of the seeds of the different genotypes studied, using a sample of 23 seeds of each individual, considering polyembryonic those with more than one embryo per seed, according to methodology described Santos, Girardi, Vieira, Ledo, and Soares Filho (2015).

\section{Results and Discussion}

In this study, the WPM resulted in positive responses, once the the explants of all genotypes showed direct regeneration and formed plants. In the micropropagation of the different citrus genotypes considered (Figure 1, Table 1). The genotypes that formed the largest number of plants from the explants grown in vitro were the 'Indio' mean number of explants obtained per plant (EP) and total number of plants obtained (TP) of 4.2 and 279, respectively, 'Riverside' (EP 2.8 and TP 110) and 'San Diego' (EP 3.0 and TP 118) citrandarins, and the hybrids $\mathrm{CSM} \times(\mathrm{RL} \times \mathrm{TR})-059(\mathrm{EP} 3.6$ and TP 203) and CSM $\times$ TRBK Colombia (EP 3.6 and TP 128). The results were 
lower for the hybrids TRH-051 (EP 2.0 and TP 71), TRH-069 (EP 1.4 and TP 67), FRL $\times($ RL $\times$ TR)-005 (EP 1.0 and TP 66), RL $\times$ TR-001 (EP 1.0 and TP 62) and 'Sunki Tropical' mandarin (EP 1.0 and TP 25).

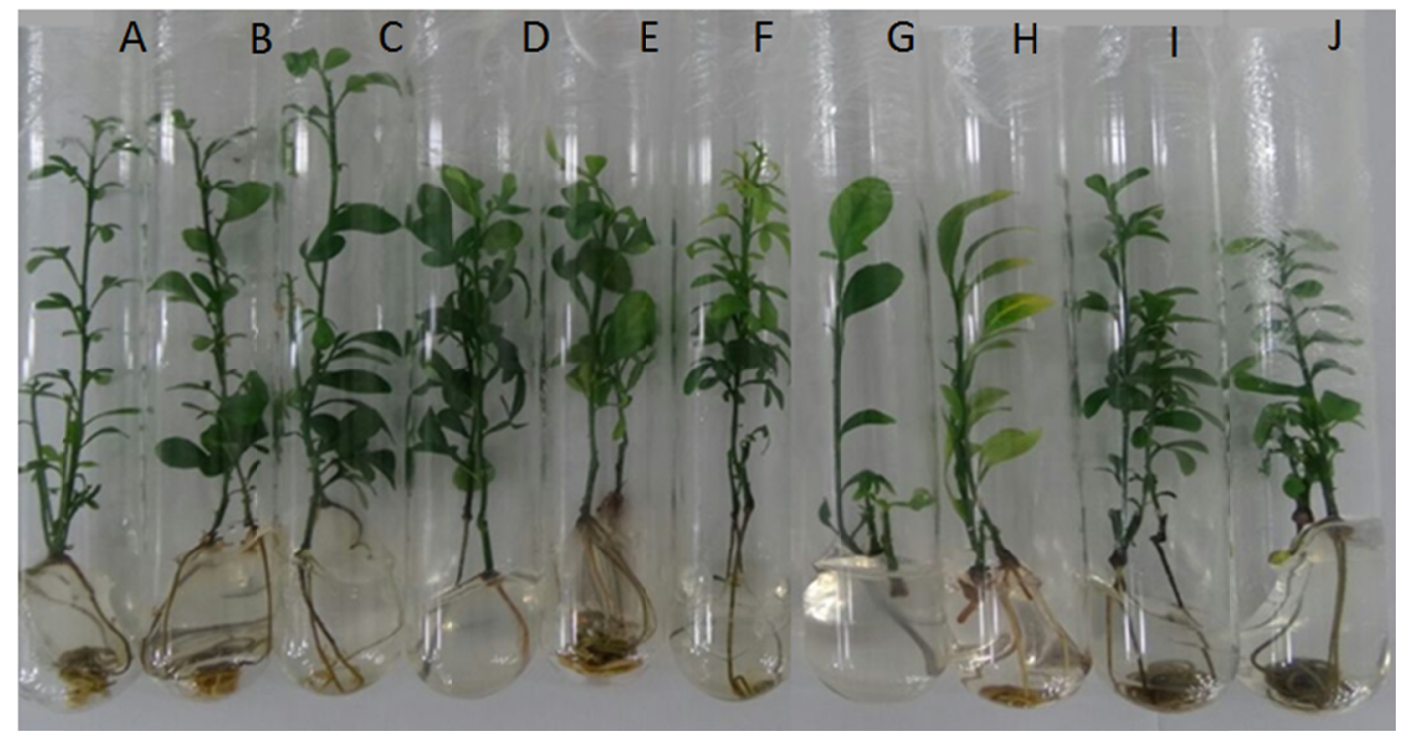

Figure 1. Plants obtained from explants cultured in vitro in Wood Plant Medium (WPM), related to the genotypes RL $\times$ TR-001 (A), 'San Diego' (B), 'Indio' (C) and Riverside (D) citrandarins [Citrus sunki (Hayata) hort. ex Tanaka $\times$ Poncirus trifoliata (L.) Raf.], FRL $\times($ RL $\times$ TR)-005 (E), TRH-051 (F), TRH-069 (G), CSM $\times($ RL $\times$ TR)-059 (H), CSM $\times$ TRBK Colombia (I) and 'Sunki Tropical' (C. sunki) mandarin (J), where RL = 'Rangpur' lime (C. limonia Osbeck), TR $=P$. trifoliata, $\mathrm{FRL}=$ 'Florida' rough lemon ( . jambhiri Lush. $)$, $\mathrm{TRH}=$ trifoliate hybrid (involving $P$. trifoliata), CSM = common 'Sunki' $(C$. sunki) mandarin, TRBK $=$ P. trifoliata 'Benecke'

Table 1. Number of mother plants, number of explants (nodal segments with approximate length of $1 \mathrm{~cm}$ ), mean number of explants produced per plant and total number of plants obtained after culturing different citrus genotypes for 180 days in Wood Plant Medium (WPM)

\begin{tabular}{lllll}
\hline Genotypes $^{1}$ & Mother plants & Explants & Mean explants per plant ${ }^{2}$ & Total plants obtained $^{-}$ \\
\hline 'Indio' citrandarin & 10 & 42 & $4.2 \mathrm{a}$ & 279 \\
'Riverside' citrandarin & 11 & 31 & $2.8 \mathrm{a}$ & 110 \\
'San Diego' citrandarin & 10 & 30 & $3.0 \mathrm{a}$ & 118 \\
'Sunki Tropical' mandarin & 6 & 6 & $1.0 \mathrm{~b}$ & 25 \\
$\mathrm{RL} \times \mathrm{TR}-001$ & 8 & 8 & $1.0 \mathrm{~b}$ & 62 \\
FRL $\times(\mathrm{RL} \times \mathrm{TR})-005$ & 11 & 11 & $1.0 \mathrm{~b}$ & 66 \\
$\mathrm{CSM} \times(\mathrm{RL} \times \mathrm{TR})-059$ & 11 & 39 & $3.6 \mathrm{a}$ & 203 \\
TRH-051 & 8 & 16 & $2.0 \mathrm{~b}$ & 71 \\
TRH-069 & 10 & 14 & $1.4 \mathrm{~b}$ & 67 \\
$\mathrm{CSM} \times$ TRBK Colombia & 11 & 39 & $3.6 \mathrm{a}$ & 128 \\
\hline
\end{tabular}

Note. 'Indio', 'Riverside' and 'San Diego' citrandarins [Citrus sunki (Hayata) hort. ex Tanaka $\times$ Poncirus trifoliata (L.) Raf.]; 'Sunki Tropical' (C. sunki) mandarin; RL: 'Rangpur' lime (C. limonia Osbeck); TR: $P$. trifoliata; FRL: 'Florida' rough lemon (C. jambhiri Lush.); CSM: common 'Sunki' (C. sunki) mandarin; TRH: trifoliate hybrid (involving P. trifoliata); TRBK: P. trifoliata 'Benecke'.

Tallón et al. (2013) using adult explants of citrus rootstocks, obtained unsatisfactory results with MS medium, while WPM produced the highest percentage of explants recovered and number of buds regenerated by explants. The number of nodal segments or buds is a very important variable in micropropagation. In this experiment, it was possible to obtain a satisfactory number of nodal segments in most of the genotypes cultivated in the WPM medium at its basal concentration, even when compared to the number of buds obtained in other studies. Esmaeilnia and Dehestani (2015) obtained an average of 1.2 buds by cultivating in vitro apical segments of $C$. 
sinensis (L.) Osbeck, also in WPM medium. Oliveira, Costa, Silva, and Otoni (2010) obtained the same average of buds in the in vitro cultivation of 'Pera', 'Valencia' and 'Bahia' orange trees in WPM medium.

With respect to the 'Indio', 'Riverside' and 'San Diego' citrandarins and the hybrids CSM $\times(R L \times T R)-059$ and $\mathrm{CSM} \times$ TRBK Colombia, the good performance of WPM in the micropropagation coincides with the results obtained by Tallón et al. (2013) utilizing explants from adult plants of 'Alemow' (C. macrophylla Wester) and 'Sour' orange (Citrus $\times$ aurantium L.), for which WPM produced a higher percentage of explants recovered and number of shoots regenerated per explant than did MS medium.

In this study, we obtained a satisfactory number of nodal segments from five of the ten genotypes cultured in WPM medium, considering its basal concentration. Therefore, WPM can be used for micropropagation of citrus rootstocks, although adjustments are necessary to optimize the effect in relation to determined genotypes, such as 'Sunki Tropical' mandarin, for which it presented lower efficiency. These data indicate to a response at the genotype level, since the explants presented different answers in the same culture medium. This shows the fact that the conditions for the development of a species can vary among genotypes, as reported by Grattapaglia and Machado (1998), since each species or clone presents unique characteristics, determined by genetic factors. In general, we can say that WPM is a suitable medium for cloning citrus varieties, because it has been used for in vitro establishment of hundreds of citrus accessions (Carvalho et al., 2016), involving various Citrus species and related genera, such as Poncirus (Raf.), Fortunella (Swingle), Microcitrus (Swingle) and Eremocitrus (Swingle).

An important aspect to observe is the fact that the explants used in this study came from seedlings produced by germination of seeds. In this context, the polyembryony rate of the seeds of the genotypes analyzed was variable (Table 2). The 'Indio', 'Riverside' and 'San Diego' citrandarins, the 'Sunki Tropical' mandarin and the hybrids $\mathrm{TRH}-051, \mathrm{FRL} \times(\mathrm{RL} \times \mathrm{TR})-005$ and RL $\times$ TR-001 had high polyembryony rates, generally greater than $80 \%$, while the hybrids $\mathrm{CSM} \times(\mathrm{RL} \times \mathrm{TR})-059$, TRH-069 and CSM $\times$ TRBK Colombia produced seeds with moderate polyembryony rates, so the frequency of zygotic embryos (those whose genetic constitution is different than the rootstocks intended for micropropagation) was significant. Even in the case of rootstocks whose seeds have high polyembryony percentages, the occurrence of zygotic embryos is expected, of course in smaller proportions. The determination of the polyembryony rate indicates which genotypes will produce seedlings with higher or lower frequency of individuals having nucellar origin, i.e., that have the same genetic constitution of the mother plant. It is known that the frequency of individuals with nucellar origin is directly proportional to the polyembryony rate of the seeds of the variety utilized (Soares Filho et al., 2014).

Therefore, WPM in its basal composition is efficient for propagation both from seeds with nucellar origin and zygotic origin, which reinforces the possibility of its use in micropropagation of genotypes with different genetic constitutions. We are planning to perform molecular analyses of the seedlings investigated in this study, to identify the individuals with nucellar origin for employment in subsequent micropropagation, while discarding those with hybrid origin.

Table 2. Polyembryony rate of the citrus genotypes utilized

\begin{tabular}{ll}
\hline Genotypes $^{1}$ & Polyembryony rate (\%) \\
\hline 'Indio' citrandarin & 91 \\
'Riverside' citrandarin & 96 \\
'San Diego' citrandarin & 78 \\
'Sunki Tropical' mandarin & 87 \\
RL $\times$ TR-001 & 96 \\
FRL $\times($ RL $\times$ TR)-005 & 84 \\
CSM $\times(R L \times$ TR)-059 & 58 \\
TRH-051 & 87 \\
TRH-069 & 65 \\
CSM $\times$ TRBK Colombia & 55
\end{tabular}

Note. 'Indio', 'Riverside' and 'San Diego' citrandarins [Citrus sunki (Hayata) hort. ex Tanaka $\times$ Poncirus trifoliata (L.) Raf.]; 'Sunki Tropical' (C. sunki) mandarin; RL: 'Rangpur' lime (C. limonia Osbeck); TR: P. trifoliata; FRL: 'Florida' rough lemon (C. jambhiri Lush.); CSM: common 'Sunki' (C. sunki) mandarin; TRH: trifoliate hybrid (involving P. trifoliata); TRBK: P. trifoliata 'Benecke'. 
An additional advantage is the convenience of using explants from nodal segments of adult plants, i.e., already in the fruit-bearing stage, free of pests and corresponding to the varieties targeted for micropropagation. This avoids the need to identify and discard seedlings with hybrid nature (zygotic), as found in this study, besides using individuals in the non-juvenile phase (in the case of micropropagation of rootstocks), to enable start of fruit production from the scion varieties grafted on them sooner than would be the case of juvenile rootstocks, a common situation in the traditional method of obtaining rootstocks from seeds.

In this sense, it can be observed that the use of the in vitro culture using WPM culture medium in its basal constitution, proved to be an important strategy to propagate citrus rootstocks of agronomic interest. In the case of citrus, this technique is a possibility for the propagation of different genotypes, as an alternative to the multiplication of plants only by means of seeds.

\section{Conclusion}

The WPM medium was suitable for micropropagation of the citrus genotypes studied, with the 'Indio' citrandarin and the hybrids CSM x (RL x TR)-059 and CSM x TRBK Colombia standing out as the best performers.

\section{Acknowledgments}

To the Coordination of Improvement of Higher Education Personnel (CAPES) for the granting of a master's degree scholarship to the first author, the Embrapa Cassava \& Tropical Fruits and to the National Council for Scientific and Technological Development $(\mathrm{CNPq})$ for the financial assistance.

\section{References}

Carvalho, M. de J. da S de., Souza, A. da S., Santos, E. B., Soares Filho, W. dos S., Ledo, C. A. da S., \& Souza, F. V. D. (2016). Univariate and multivariate statistical tools for in vitro conservation of citrus genotypes. Acta Scientiarum Agronomy, 38, 129-137. https://doi.10.4025/actasciagron.v38i1.26433

Cervera, M., Navarro, A., Navarro, L., \& Peña, L. (2008). Production of transgenic adult plants from clementine mandarin by enhancing cell competence for transformation and regeneration. Tree Physiology, 28, 55-66. https://doi.org/10.1093/treephys/28.1.55

Engelmann, F. (2011). Use of biotechnologies for the conservation of plant biodiversity. In Vitro Cellular \& Developmental Biology-Plant, 47, 5-16. https://doi.10.1093/treephys/28.1.55

Esmaeilnia, E., \& Dehestani, A. (2015). In vitro plant regeneration from mature tissues of Thomson navel sweet orange (Citrus sinensis L. Osbeck.). Biharean Biologist, 9, 9-14.

Grattapaglia, D., \& Machado, M. A. (1998). Micropropagação. In A. C. Torres, L. S. Caldas, \& J. A. Buso (Eds.), Cultura de tecidos e transformação genética de plantas (Vol. 1, pp. 183-260). Brasília: Embrapa-SPI/ Embrapa-CNPH.

Lloyd, G., \& McCown, B. (1980). Use of microculture for production and improvement of Rhododendron spp. Horticultural Science, 15, 416-417.

Murashige, T., \& Skoog, F. (1962). A revised medium for rapid growth and bioassays with tobacco tissue cultures. Physiologia Plantarum, 15, 473-497. https://doi.org/10.1111/j.1399-3054.1962.tb08052.x

Oliveira, M. L. P., Costa, M. G. C., Silva, C. V. da, \& Otoni, W. C. (2010). Growth regulators, culture media and antibiotics in the in vitro shoot regeneration of mature tissue of citrus cultivars. Pesquisa Agropecuária Brasileira, 45, 654-660. https://doi.org/10.1590/S0100-204X2010000700004

Ruzic, D., Saric, M., Cerovic, R., \& Culafic, L. J. (2004). Contents of macroelements and growth of sweet cherry rootstock in vitro. Biologia Plantarum, 47, 463-465. https://doi.org/10.1023/B:BIOP.0000023897.84367.41

Santos, C. Q. de J., Girardi, E. A., Vieira, E. L., Ledo, C. A. da S., \& Soares Filho, W. dos S. (2015). Optimum sample size of fruits and seeds for polyembryony determination in citrus. Revista Brasileira de Fruticultura 37, 172-178. https://doi.org/10.1590/0100-2945-063/14

Sharma, S., Prakash, A., \& Tele, A. (2009). In vitro propagation of citrus rootstocks. Notulae Botanicae Horti Agrobotanici Cluj-Napoca, 37, 84-88.

Soares Filho, W. dos S., Souza, U., Ledo, C. A. da S., Santana. L. G. L., \& Passos, O. S. (2014). Polyembryony e potencial de obtenção de hybrids em citros. Revista Brasileira de Fruticultura, 36, 950-956. https://doi.org/10.1590/0100-2945-345/13 
Tallón, C. I., Porras, I., \& Pérez, T. O. (2013). High efficiency in vitro organogenesis from mature tissue explants of Citrus macrophylla and C. aurantium. In Vitro Cellular \& Developmental Biology-Plant, 49, $145-155$. https://doi.org/10.1007/s11627-012-9476-6

\section{Copyrights}

Copyright for this article is retained by the author(s), with first publication rights granted to the journal.

This is an open-access article distributed under the terms and conditions of the Creative Commons Attribution license (http://creativecommons.org/licenses/by/4.0/). 